\title{
Morphological analysis of age-related iridocorneal angle changes in normal and glaucomatous cases using anterior segment optical coherence tomography
}

\author{
This article was published in the following Dove Press journal: \\ Clinical Ophthalmology \\ 21 December 2013 \\ Number of times this article has been viewed
}

\section{Yuko Maruyama \\ Kazuhiko Mori \\ Yoko Ikeda \\ Morio Ueno \\ Shigeru Kinoshita}

Department of Ophthalmology, Kyoto Prefectural University of Medicine, Kyoto, Japan
Correspondence: Kazuhiko Mori Department of Ophthalmology, Kyoto Prefectural University of Medicine, 465 Kajii-cho,

Hirokoji-agaru, Kawaramachi-dori, Kamigyo-ku, Kyoto 602-084I, Japan

Tel $+8 I 7525 I 5578$

Fax +81752515663

Email kmori@koto.kpu-m.ac.jp
Purpose: To analyze age-related morphological changes of the iridocorneal angle in normal subjects and glaucomatous cases, using anterior segment optical coherence tomography (AS-OCT).

Methods: This study involved 58 eyes of 58 open-angle glaucoma cases and 72 eyes of 72 age-matched normal-open-angle control subjects. Iridocorneal angle structures in nasal and temporal regions and anterior chamber depth (ACD) were measured using AS-OCT. Axial length and refractive error were measured by use of an ocular biometer and auto refractor keratometer. Angle opening distance (AOD), angle recess area (ARA), and trabecular-iris space area (TISA), measured at $500 \mu \mathrm{m}$ (TISA500) and $750 \mu \mathrm{m}$ (TISA750) distant from the scleral spur, were calculated, in the nasal and temporal regions. A new index, the peripheral angle frame index (PAFI), which represents the peripheral angle structure, was proposed, and was defined as (TISA750-TISA500)/TISA500.

Results: Refractive power in the glaucoma cases was less than in control cases $(P<0.0001)$. Axial length $(P<0.0001)$ and ACD $(P=0.0004)$ were longer and deeper, respectively, in the glaucoma cases, compared with the control cases. In both control and glaucoma groups, ACD, AOD, ARA, and TISA decreased linearly in an age-dependent manner, while PAFI stayed at relatively constant values throughout the age distribution. AOD in the glaucoma group was longer than in the control group, in both the temporal and nasal regions; ARA and TISA were larger in the glaucoma than in the control group. However, no significant differences in nasal or temporal PAFI were found between the glaucoma and control groups.

Conclusion: The findings of this study show that AS-OCT is useful for the quantitative evaluation of age-related changes in peripheral angle structure in glaucoma and control cases.

Keywords: iridocorneal angle structures, anterior segment optical coherence tomography, AS-OCT, peripheral angle frame index, PAFI

\section{Introduction}

Previously used methods for evaluation of the anterior chamber angle of the eye, such as slit-lamp biomicroscopy, or gonioscopy, are known to be subjective. Ultrasound biomicroscopy (UBM) reportedly allows for a quantitative measurement of the anterior chamber angle..$^{1-4}$ However, due to the fact that UBM is a contact measurement, the utilization of this method is limited to patient screening, and for immediate postoperative use. Anterior segment optical coherence tomography (AS-OCT) is a new imaging technology that reportedly provides a non-contact quantitative evaluation of the anterior segment of the eye..$^{5-12}$ The recently released Visante ${ }^{\circledR}$ OCT version 2.0.1.88 system software 
(Carl Zeiss Meditec AG, Jena, Germany) includes several features that were previously not available. Of those, an enhanced anterior segment single mode provides an image averaged from four consecutive anterior segment scans, to improve contrast. Moreover, the new version includes a semiautomated program to assess the anterior chamber angle. One advantage of using AS-OCT for assessment of the anterior chamber angle is that image acquisition and subsequent measurement can be performed under light-controlled conditions, with patients in the sitting position, and without alternation of angle structure by irregular lighting and physical contact. However, some disadvantages have also been reported, such as poor agreement between gonioscopic and AS-OCT assessment. ${ }^{7,13}$ In addition, reproducibility of the AS-OCT findings for evaluation of the anterior chamber angle has been questioned. In particular, the reproducibility of inferior quadrant findings is reported to be poor, due to variability of scleral spur placement; reproducibility of anterior chamber angle parameters in nasal and temporal angles was better than in the inferior quadrants. ${ }^{7,14,15}$

Recently, there have been many studies published that report the use of AS-OCT for assessment of the anterior segment. ${ }^{15-19}$ Nongpiur et al used AS-OCT images to evaluate anterior chamber width, defined as the horizontal scleral spur-to-spur distance, and found that it was smaller in angleclosure patients than in open-angle patients. ${ }^{20}$ Moghimi et al also found that the anterior segment was crowded in closed-angle eyes, compared with open-angle eyes. ${ }^{21}$ Several cross-sectional studies have assessed the influence of age on anterior chamber depth (ACD). ${ }^{22-24}$ However, there are few reports about age-related changes of the peripheral angle structure. ${ }^{25}$ In this study, a new index, termed the peripheral angle frame index (PAFI), was developed and proposed for representing the peripheral angle structure. PAFI is defined as the difference between trabecular-iris space area (TISA) measured at a distance of $750 \mu \mathrm{m}$ (TISA750) and TISA measured at a distance of $500 \mu \mathrm{m}$ (TISA500), divided by TISA500. We used this index to investigate how peripheral angles changed with age. The TISA parameter was used instead of the angle recess area (ARA) because it has been proposed that ARA may be less sensitive for identifying narrow angles in eyes with deep angle recess. ${ }^{26-28}$ The aim of this study was to analyze the cross-sectional, age-related morphological changes of the iridocorneal angle in normal subjects, and in open-angle glaucoma patients, using AS-OCT.

\section{Materials and methods}

This study involved 58 eyes of 58 open-angle glaucoma patients ( 35 females, 23 males) and 72 eyes of 72 age-matched, normal open-angle, volunteer control subjects (49 females, 23 males), diagnosed as non-glaucomatous by glaucoma specialists at the Glaucoma Clinic of Kyoto Prefectural University of Medicine, Kyoto, Japan, from 2007 to 2009. All 130 subjects included in the study were of Japanese race. The diagnostic criteria for normal-tension glaucoma (NTG) were 1) normal iridocorneal open angle, 2) no evidence of high intraocular pressure (IOP) (IOP $\leq 21 \mathrm{mmHg}$ ), 3 ) glaucomatous changes in the visual field, with optic nerve cupping, and 4) absence of other optic neuropathies. For the diagnosis of primary open-angle glaucoma (POAG), the criteria were the same as with (1), (3), and (4) above, but with a maximum IOP $>21 \mathrm{mmHg}$. Both NTG and POAG were diagnosed according to the guidelines of the Japan Glaucoma Society ${ }^{29}$ and the European Glaucoma Society. ${ }^{30}$

The normal open-angle control subjects comprised individuals with IOP $\leq 21 \mathrm{mmHg}$ in both eyes, as measured by Goldmann applanation tonometry with normal optic discs, with open angles on gonioscopy, and with no suspicion of any form of glaucoma. When subjects exhibited a narrow angle equal to grade 2 or less, as determined using the Van Herick system, they were excluded from the study. ${ }^{31}$

Informed consent was obtained from all participants prior to their involvement in the study, in accordance with the tenets set forth in the Declaration of Helsinki.

In all eyes, Visante OCT was used in the enhanced highresolution mode ("high-resolution corneal" protocol) to measure iridocorneal angle structures (Figure 1A-C), and in the enhanced anterior segment mode ("enhanced anterior segment single" protocol) to measure ACD (Figure 1D). AS-OCT images were obtained in darkroom lighting conditions.

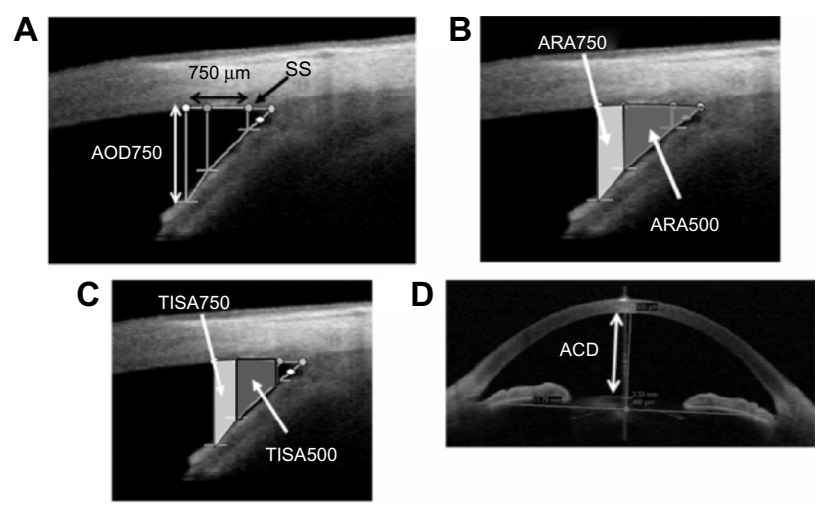

Figure I Anterior segment optical coherence tomography images. Notes: Iridocorneal angle structures measured by use of the enhanced high resolution mode of anterior segment optical coherence tomography. The images show (A) AOD750, (B) ARA500/750, (C) TISA500/750, and (D) ACD.

Abbreviations: SS, scleral spur; AOD750, anterior opening distance at $750 \mu \mathrm{m}$; ARA500/750, angle recess area at 500/750 $\mu \mathrm{m}$; TISA500/750, trabecular-iris space area at 500/750 $\mu \mathrm{m} ; A C D$, anterior chamber depth. 
The enhanced anterior segment mode image was obtained first, and then nasal-side and temporal-side images were obtained using the enhanced high resolution mode. Images were captured at the nasal and temporal angle quadrants (3- and 9-o'clock meridians; nasal-temporal angles at $\left.0^{\circ}-180^{\circ}\right)$. Internal fixation was used in all subjects. With each subject, the procedure was performed first on the right eye, and then on the left eye. Axial length and refractive error were measured using the IOL Master (Carl Zeiss Meditec AG), and the RKT-7700 autorefractor keratometer (Nidek, Gamagori, Japan), respectively. Angle-opening distance (AOD), at $500 \mu \mathrm{m}$ and $750 \mu \mathrm{m}$ distant from the scleral spur (AOD500 and AOD750, respectively) (Figure 1A), ARA at $500 \mu \mathrm{m}$ and $750 \mu \mathrm{m}$ distant (ARA500 and ARA750, respectively) (Figure 1B), and TISA at $500 \mu \mathrm{m}$ and $750 \mu \mathrm{m}$ distant (TISA500 and TISA750, respectively) (Figure 1C), were automatically calculated, in the nasal and temporal regions of the right eye of each subject, using Visante OCT device software. When the right eye could not be evaluated, due to extended measurement time required to capture the image, the left eye was used. Right eye data was used whenever possible. Subjects of whom the images produced were of inadequate quality, in whom there was difficulty in detecting the scleral spur, or in whom there had been previous surgical intervention that affected angle structure (such as cataract surgery or laser iridotomy), were excluded from the study.

Iridocorneal angle structures, axial length, and refractive error, in the nasal and temporal regions, were then compared between glaucoma patients against normal control subjects. The Student's $t$-test was used for statistical analysis. The slopes of the iridocorneal structural parameters were derived, to assess age-dependent differences, using a linear mixed effect model. Based on the absolute slopes of the various AS-OCT parameters, normalized slopes were calculated, as absolute slope divided by mean parameter value, in order to compare relative rates of change of all parameters. ${ }^{25}$ A $P$-value lower than 0.05 was considered statistically significant.

\section{Results}

The mean ages of the 58 open-angle glaucoma patients (POAG: 20 cases, NTG: 38 cases) and 72 age-matched normal control subjects were $59.5 \pm 13.8$ years and $62.1 \pm 12.8$ years, respectively. Refractive power in the glaucoma group was lower than in control subjects $(P<0.0001)$. Axial length $(P<0.0001)$ and $\operatorname{ACD}(P=0.0004)$ were longer and deeper, respectively, in glaucoma cases, compared with control subjects (Table 1).
Table I Comparison of demographic and examination data between glaucoma patients and normal controls

\begin{tabular}{llll}
\hline & $\begin{array}{l}\text { Glaucoma } \\
(\mathbf{n}=\mathbf{5 8})\end{array}$ & $\begin{array}{l}\text { Control } \\
(\mathbf{n}=\mathbf{7 2})\end{array}$ & P-value \\
\hline Age (years) & $59.5 \pm I 3.8$ & $62.1 \pm I 2.8$ & 0.26 \\
Male:female & $23: 35$ & $23: 49$ & 0.46 \\
Right:left & $33: 25$ & $53: 19$ & 0.062 \\
Refractive power (D) & $-3.90 \pm 4.46$ & $0.49 \pm 3.12$ & $<0.000 \mathrm{I}^{\mathrm{a}}$ \\
Axial length (mm) & $25.9 \pm 2.6$ & $24.1 \pm I .7$ & $<0.000 \mathrm{I}^{\mathrm{a}}$ \\
ACD (mm) & $2.96 \pm 0.40$ & $2.74 \pm 0.30$ & $0.0004^{\mathrm{a}}$ \\
\hline
\end{tabular}

Notes: a $P<0.05$ (independent samples $t$-test between glaucoma patients and normal controls). The values presented for age, refractive power, axial length and $A C D$ are mean \pm standard deviation.

Abbreviation: $A C D$, anterior chamber depth.

No differences in angle structure were observed between nasal or temporal regions in both glaucoma cases and control subjects. However, ACD $(P=0.0004)$ (Table 1) and all peripheral angle parameter values $(\mathrm{ACD}, \mathrm{AOD}, \mathrm{ARA}$, and TISA, but not PAFI) were higher in glaucoma cases than in control subjects (Table 2).

Table 3 shows the effect of age on AS-OCT parameters. All parameters (except PAFI) decreased linearly in an agedependent manner in control subjects and, in nearly the same manner, in glaucoma cases. Of these parameters, the steepest slope was shown by AOD750, of temporal sectors (slope constant: $-0.00992 \mathrm{~mm} / \mathrm{year}$, for glaucoma patients; and $-0.00867 \mathrm{~mm} /$ year, for control subjects). The representative scatter plot of AOD750 measurement at the temporal sector is shown in Figure 2.

In this study, a normalized slope was used to compare all parameters. Normalized PAFI slope values were less than $-0.005 \mathrm{au} /$ year in measurements of both nasal and temporal regions, in both glaucoma and normal control subjects. In comparison with other peripheral angle parameters, in both groups, PAFI values were found to stay relatively constant throughout the age distribution (Table 3). Moreover, among all iridocorneal parameters, only PAFI showed no differences between glaucoma cases and normal control subjects (Table 3). Van Herick measurement was used in the temporal side; thus, the temporal-side PAFI shown is representative. As shown also in Figure 3, PAFI values stayed relatively constant throughout the age distribution, in both glaucoma and control groups.

\section{Discussion}

It is well known that many glaucoma patients are myopic and have a longer axial length. In this study, we found significant myopia-related differences in refractive error, axial length, and ACD in glaucoma patients (Table 1). In glaucoma cases and control subjects, no significant differences were found 
Table 2 Comparison of angle structure between glaucoma patients and normal controls

\begin{tabular}{|c|c|c|c|c|c|c|c|c|}
\hline & \multicolumn{2}{|c|}{ Glaucoma $(n=58)$} & \multirow{2}{*}{$\frac{P \text {-value }}{\text { T vs } N}$} & \multicolumn{2}{|c|}{ Control (n=72) } & \multicolumn{3}{|c|}{$P$-value } \\
\hline & $\begin{array}{l}\text { Temporal } \\
\text { (mean } \pm \text { SD) }\end{array}$ & $\begin{array}{l}\text { Nasal } \\
\text { (mean } \pm \text { SD) }\end{array}$ & & $\begin{array}{l}\text { Temporal } \\
\text { (mean } \pm \text { SD) }\end{array}$ & $\begin{array}{l}\text { Nasal } \\
\text { (mean } \pm \text { SD) }\end{array}$ & T vs $\mathbf{N}$ & G vs $\mathbf{C}-\mathbf{T}^{\mathrm{a}}$ & G vs $\mathbf{C}-\mathbf{N}^{\mathbf{a}}$ \\
\hline Angular A (degrees) & $47.3 \pm 11.7$ & $44.8 \pm I I .5$ & 0.25 & $39.2 \pm 11.3$ & $37.5 \pm 12.3$ & 0.40 & $<0.0001$ & 0.0008 \\
\hline AOD500 $(\mathrm{mm})$ & $0.60 \pm 0.25$ & $0.54 \pm 0.22$ & 0.21 & $0.44 \pm 0.20$ & $0.42 \pm 0.20$ & 0.49 & $<0.0001$ & 0.0008 \\
\hline AOD750 (mm) & $0.82 \pm 0.31$ & $0.75 \pm 0.26$ & 0.18 & $0.6 I \pm 0.25$ & $0.58 \pm 0.24$ & 0.43 & $<0.0001$ & 0.0002 \\
\hline ARA500 $\left(\mathrm{mm}^{2}\right)$ & $0.26 \pm 0.11$ & $0.24 \pm 0.10$ & 0.23 & $0.20 \pm 0.08$ & $0.20 \pm 0.09$ & 0.42 & 0.0006 & 0.0055 \\
\hline ARA750 $\left(\mathrm{mm}^{2}\right)$ & $0.44 \pm 0.18$ & $0.40 \pm 0.15$ & 0.21 & $0.34 \pm 0.13$ & $0.32 \pm 0.14$ & 0.41 & 0.0002 & 0.0016 \\
\hline TISA500 $\left(\mathrm{mm}^{2}\right)$ & $0.22 \pm 0.09$ & $0.20 \pm 0.08$ & 0.23 & $0.17 \pm 0.06$ & $0.16 \pm 0.07$ & 0.46 & 0.0003 & 0.0030 \\
\hline TISA750 (mm²) & $0.40 \pm 0.16$ & $0.36 \pm 0.14$ & 0.21 & $0.30 \pm 0.12$ & $0.28 \pm 0.12$ & 0.44 & 0.0001 & 0.0011 \\
\hline PAFI (au/year) & $0.82 \pm 0.09$ & $0.82 \pm 0.11$ & 0.98 & $0.79 \pm 0.14$ & $0.79 \pm 0.14$ & 0.91 & 0.13 & 0.19 \\
\hline
\end{tabular}

Note: ${ }^{a}<0.05$ (independent samples $t$-test between glaucoma patients and normal controls), all except PAFI.

Abbreviations: T vs N, temporal versus nasal; G vs C-T, glaucoma patients versus normal controls in temporal regions; $\mathrm{G}$ vs C-N, glaucoma patients versus normal controls in nasal regions; AOD500/750, angle-opening distance at 500/750 $\mu \mathrm{m}$; ARA500/750, angle recess area at 500/750 $\mu \mathrm{m}$; TISA500/750, trabecular-iris space area at 500/750 $\mu \mathrm{m}$; PAFI, peripheral angle frame index; SD, standard deviation; au, arbitrary unit.

in all peripheral parameters (AOD, ARA, and TISA), in temporal and nasal regions. However, when those parameters were compared between glaucoma and control subjects, all values, except PAFI, were found to be higher in glaucoma cases (Table 2), reflecting that glaucoma patients had more severe myopia.

ACD, AOD, ARA, and TISA were found to decrease linearly in an age-dependent manner in both glaucoma cases and control subjects, mirroring the findings of previous reports about age-related decreases in ACD, ARA, and TISA in Asian people. ${ }^{18,25}$ However, to the best of our knowledge, ours is the first study to report upon age-related changes in ACD, ARA, and TISA in glaucomatous Asian patients.

In this study, a novel index, PAFI, was used in order to investigate how peripheral configuration changes with age.
The findings of a previous study show that ACD decreases with age, due to age-related lens thickening. ${ }^{21}$ Thus, the extent of cataract may affect peripheral configuration. Yet, we had no knowledge about how this affects peripheral angle structure. Moreover, we also wanted to know how changes in iris structure affect angle structure (because iris configuration changes with age due to muscle weakness and tension of the iris). Hence, we measured peripheral length parameters at $500 \mu \mathrm{m}$ and $750 \mu \mathrm{m}$ distant from the scleral spur. Being able to compare the values obtained at $500 \mu \mathrm{m}$ and $750 \mu \mathrm{m}$ distance from the scleral spur, with participants of various ages, we could elucidate age-related changes in these values. If AOD500 decreases more severely with age than AOD750, it indicates that the anterior chamber is shallower from the more peripheral area. However, if AOD500 and AOD750

Table 3 Absolute and normalized slopes of anterior segment parameters in glaucoma and control subjects

\begin{tabular}{|c|c|c|c|c|c|c|}
\hline & \multicolumn{3}{|c|}{ Glaucoma $(n=58)$} & \multicolumn{3}{|c|}{ Control $(n=72)$} \\
\hline & A-slope & N-slope & $\boldsymbol{R}$ & A-slope & N-slope & $\boldsymbol{R}$ \\
\hline $\mathrm{ACD}$ (mm/year) & -0.00599 & -0.00202 & -0.329 & $-0.00 \mid 48$ & -0.00540 & -0.429 \\
\hline Temporal AOD500 (mm/year) & -0.00599 & -0.00998 & -0.387 & -0.00742 & -0.0169 & -0.480 \\
\hline Temporal AOD750 (mm/year) & -0.00992 & -0.0121 & -0.580 & -0.00867 & -0.0142 & -0.504 \\
\hline Temporal ARA500 (mm²/year) & -0.00270 & -0.0104 & -0.333 & -0.00200 & -0.0100 & -0.334 \\
\hline Temporal ARA750 (mm²/year) & -0.00415 & -0.00943 & -0.350 & -0.00453 & -0.0133 & -0.414 \\
\hline Temporal TISA500 (mm²/year) & -0.00214 & -0.00972 & -0.321 & -0.00197 & -0.0116 & -0.388 \\
\hline Temporal TISA750 (mm²/year) & -0.00413 & -0.0103 & -0.344 & -0.00397 & -0.0142 & -0.447 \\
\hline Temporal PAFI (au/year) & -0.000381 & -0.000465 & -0.258 & -0.00356 & $-0.0045 \mid$ & -0.316 \\
\hline Nasal AOD500 (mm/year) & -0.00630 & -0.0117 & -0.355 & -0.00557 & -0.0133 & $-0.4 I I$ \\
\hline Nasal AOD750 (mm/year) & -0.00809 & -0.0108 & -0.422 & -0.00967 & -0.0167 & -0.519 \\
\hline Nasal ARA500 ( $\mathrm{mm}^{2} /$ year $)$ & -0.00199 & -0.00829 & -0.279 & -0.00229 & -0.0115 & -0.340 \\
\hline Nasal ARA750 (mm²/year) & -0.00369 & -0.00922 & -0.330 & -0.00203 & -0.00634 & -0.398 \\
\hline Nasal TISA500 (mm²/year) & -0.00178 & -0.00890 & -0.309 & -0.00203 & -0.0127 & -0.366 \\
\hline Nasal TISA750 (mm²/year) & -0.00347 & -0.00964 & -0.350 & -0.00397 & -0.0142 & -0.418 \\
\hline Nasal PAFI (au/year) & -0.00119 & -0.00145 & -0.252 & -0.00377 & -0.00477 & -0.343 \\
\hline
\end{tabular}

Abbreviations: $R$, correlation coefficient; A-slope, absolute slope; N-slope, normalized slope; ACD, anterior chamber depth; AOD500/750, angle-opening distance at $500 / 750 \mu \mathrm{m}$; ARA500/750, angle recess area at 500/750 $\mu \mathrm{m}$; TISA500/750, trabecular-iris space area at 500/750 $\mu \mathrm{m}$; PAFI, peripheral angle frame index; au, arbitrary unit. 


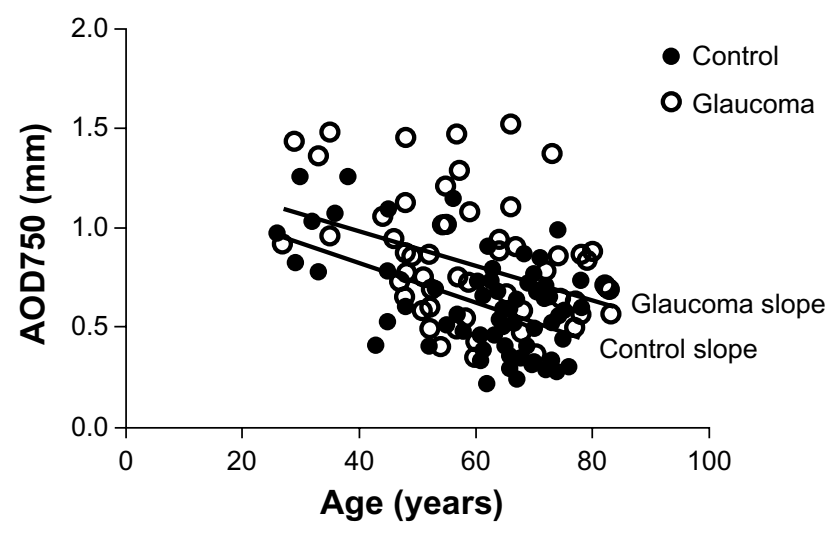

Figure 2 Relationship between temporal AOD750 and subject age in glaucoma and normal subjects.

Notes: Scatter plot showing distribution of temporal AOD750, determined by anterior segment optical coherence tomography. The lines show absolute slope in both glaucoma and control groups.

Abbreviation: AOD750, angle opening distance at $750 \mu \mathrm{m}$.

decrease in a similar manner, it indicates that the anterior chamber area may decrease at the same rate as the peripheral area, as in a more central part of the peripheral region. Thus, we posit that using this new index will help in understanding changes of the peripheral angle. A normalized slope was used to compare all parameters; PAFI was found to stay within relatively constant values throughout the age distribution, in both the glaucoma and control groups (Table 3). These findings suggest that the peripheral area will decrease at the same rate with age. In addition, PAFI was the only parameter with no difference found between the glaucoma and control groups (Table 3), as no age-related change of the peripheral angle structure was found in either group.

Ideally, the effect of age on ACD and other peripheral angle parameters is best studied by means of longitudinal

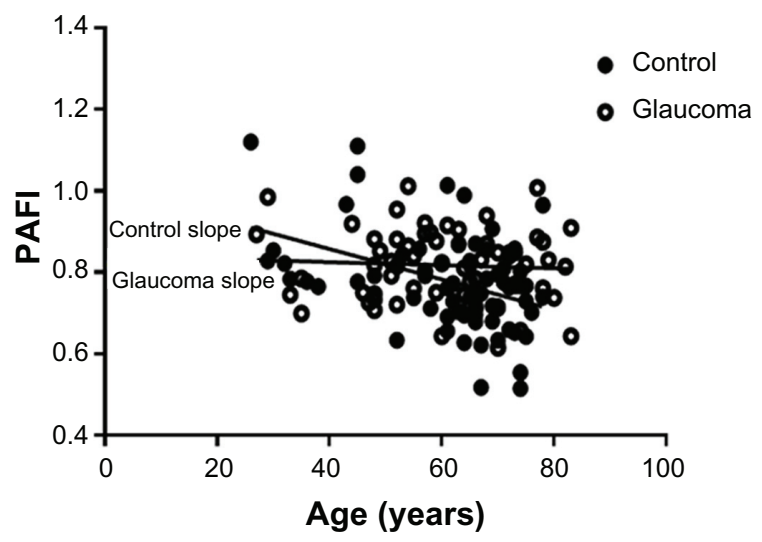

Figure 3 Relationship between PAFI and subject age in glaucoma and normal subjects.

Notes: The scatter plot showing distribution of temporal PAFI, determined by AS-OCT. The lines show absolute slope in both glaucoma and control groups. PAFI was found to stay within relatively constant values throughout the age distribution. Abbreviation: PAFI, peripheral angle frame index; AS-OCT, anterior segment optical coherence tomography. follow-up measurements, to determine whether aging people do indeed show gradual reduction in parameters based on quantitative measures, such as AS-OCT. Further study is needed to verify long-term changes of peripheral parameters.

It should be noted that this study has some limitations. First, this was a clinic-based study, not a population-based study. Second, there may have been some selection bias in regard to the samples, as glaucoma subjects were recruited from the glaucoma clinic of our university hospital.

It should also be noted that since all of the subjects involved in this study were Japanese, the results may not be applicable to other racial groups. Moreover, only open-angle participants were analyzed in this study; the results might differ from those obtained with other types of glaucoma or control subjects with shallow eyes. In addition, subjects who had undergone cataract surgery were excluded from the study, so that most parameters were measured with the participants own lens; the extent of cataract may have affected the results.

Finally, although AS-OCT was found to be useful, its use is associated with some limitations. For example, in many cases, data cannot be analyzed, due to inability to find the sclera spur. ${ }^{14,32,33}$ In this study, only temporal-region and nasal-region data was used, as it is reportedly extremely difficult to obtain reproducible images in the superior and inferior regions. ${ }^{15}$ It is hoped that in the future, AS-OCT devices will be able to provide improved, higher-quality images, and that peripheral angle parameters can be compared with other biometrics. ${ }^{34}$

In summary, the findings of this study show that AS-OCT is useful for the quantitative evaluation of age-related changes of the peripheral angle structure.

\section{Disclosure}

The authors report no conflicts of interest associated with this work.

\section{References}

1. Pavlin CJ, Foster FS. Ultrasound biomicroscopy. High-frequency ultrasound imaging of the eye at microscopic resolution. Radiol Clin North Am. 1998;36(6):1047-1058.

2. Sakata LM, Wong TT, Wong HT, et al. Comparison of Visante and slit-lamp anterior segment optical coherence tomography in imaging the anterior chamber angle. Eye (Lond). 2010;24(4):578-587.

3. Urbak SF, Pedersen JK, Thorsen TT. Ultrasound biomicroscopy. II. Intraobserver and interobserver reproducibility of measurements. Acta Ophthalmol Scand. 1998;76(5):546-549.

4. Tello C, Liebmann J, Potash SD, Cohen H, Ritch R. Measurement of ultrasound biomicroscopy images: intraobserver and interobserver reliability. Invest Ophthalmol Vis Sci. 1994;35(9):3549-3552. 
5. Grulkowski I, Gora M, Szkulmowski M, et al. Anterior segment imaging with Spectral OCT system using a high-speed CMOS camera. Opt Express. 2009;17(6):4842-4858.

6. Garcia JP Jr, Rosen RB. Anterior segment imaging: optical coherence tomography versus ultrasound biomicroscopy. Ophthalmic Surg Lasers Imaging. 2008;39(6):476-484.

7. Sakata LM, Lavanya R, Friedman DS, et al. Comparison of gonioscopy and anterior segment ocular coherence tomography in detecting angle closure in different quadrants of the anterior chamber angle. Ophthalmology. 2008;115(5):769-774.

8. Dada T, Sihota R, Gadia R, Aggarwal A, Mandal S, Gupta V. Comparison of anterior segment optical coherence tomography and ultrasound biomicroscopy for assessment of the anterior segment. J Cataract Refract Surg. 2007;33(5):837-840.

9. Leung $\mathrm{CK}$, Li H, Weinreb RN, et al. Anterior chamber angle measurement with anterior segment optical coherence tomography: a comparison between slit lamp OCT and Visante OCT. Invest Ophthalmol Vis Sci. 2008;49(8):3469-3474.

10. Bailey MD, Sinnott LT, Mutti DO. Ciliary body thickness and refractive error in children. Invest Ophthalmol Vis Sci. 2008;49(10):4353-4360.

11. Pekmezci M, Porco TC, Lin SC. Anterior segment optical coherence tomography as a screening tool for the assessment of the anterior segment angle. Ophthalmic Surg Lasers Imaging. 2009;40(4): 389-398.

12. Khor WB, Sakata LM, Friedman DS, et al. Evaluation of scanning protocols for imaging the anterior chamber angle with anterior segmentoptical coherence tomography. J Glaucoma. 2010;19(6):365-368.

13. Nolan WP, See JL, Chew PT, et al. Detection of primary angle closure using anterior segment optical coherence tomography in Asian eyes. Ophthalmology. 2007;114(1):33-39.

14. Sakata LM, Lavanya R, Friedman DS, et al. Assessment of the scleral spur in anterior segment optical coherence tomography images. Arch Ophthalmol. 2008;126(2):181-185.

15. Kim DY, Sung KR, Kang SY, et al. Characteristics and reproducibility of anterior chamber angle assessment by anterior-segment optical coherence tomography. Acta Ophthalmol. 2011;89(5):435-441.

16. Liu S, Li H, Dorairaj S, et al. Assessment of scleral spur visibility with anterior segment optical coherence tomography. J Glaucoma. 2010;19(2):132-135.

17. Tan AN, Sauren LD, de Brabander J, et al. Reproducibility of anterior chamber angle measurements with anterior segment optical coherence tomography. Invest Ophthalmol Vis Sci. 2011;52(5):2095-2099.

18. Congdon NG, Kong X, Meltzer ME, et al. Determinants and two-year change in anterior chamber angle width in a Chinese population. Ophthalmology. 2012;119(12):2500-2506.

19. Deokule S, Alencar L, Vizzeri G, Medeiros F, Weinreb RN. Comparison of unenhanced and enhanced imaging protocols for angle measurements with anterior segment optical coherence tomography. Ophthalmic Surg Lasers Imaging. 2012;43(1):39-44.
20. Nongpiur ME, Sakata LM, Friedman DS, et al. Novel association of smaller anterior chamber width with angle closure in Singaporeans. Ophthalmology. 2010;117(10):1967-1973.

21. Moghimi S, Vahedian Z, Fakhraie G, et al. Ocular biometry in the subtypes of angle closure: an anterior segment optical coherence tomography study. Am J Ophthalmol. 2013;155(4):664-673, 673. e1.

22. Rufer F, Schroder A, Klettner A, Frimpong-Boateng A, Roider JB, Erb C. Anterior chamber depth and iridocorneal angle in healthy White subjects: effects of age, gender and refraction. Acta Ophthalmol. 2010;88(8):885-890.

23. Xu L, Cao WF, Wang YX, Chen CX, Jonas JB. Anterior chamber depth and chamber angle and their associations with ocular and general parameters: the Beijing Eye Study. Am J Ophthalmol. 2008;145(5): 929-936.

24. He M, Huang W, Zheng Y, Alsbirk PH, Foster PJ. Anterior chamber depth in elderly Chinese: the Liwan eye study. Ophthalmology. 2008;115(8):1286-1290.

25. Cheon MH, Sung KR, Choi EH, et al. Effect of age on anterior chamber angle configuration in Asians determined by anterior segment optical coherence tomography; clinic-based study. Acta Ophthalmol. 2010;88(6):e205-e210.

26. Radhakrishnan S, Goldsmith J, Huang D, et al. Comparison of optical coherence tomography and ultrasound biomicroscopy for detection of narrow anterior chamber angles. Arch Ophthalmol. 2005;123(8): $1053-1059$.

27. Leung CK, Cheung CY, Li H, et al. Dynamic analysis of dark-light changes of the anterior chamber angle with anterior segment OCT. Invest Ophthalmol Vis Sci. 2007;48(9):4116-4122.

28. Hirose F, Hata M, Ito SI, Matsuki T, Kurimoto Y. Light-dark changes in iris thickness and anterior chamber angle width in eyes with occludable angles. Graefe's Arch Clin Exp Ophthalmol. May 21, 2013. [Epub ahead of print.]

29. The Japan Glaucoma Society Guidelines for Glaucoma. (3rd ed). Nihon Ganka Gakkai Zasshi. 2012;116(1):3-46.

30. European Glaucoma Society. Terminology and Guidelines for Glaucoma. 3rd ed. Savona, Italy: Dogma; 2008.

31. Van Herick W, Shaffer RN, Schwartz A. Estimation of width of angle of anterior chamber. Incidence and significance of the narrow angle. Am J Ophthalmol. 1969;68(4):626-629.

32. Lavanya R, Foster PJ, Sakata LM, et al. Screening for narrow angles in the singapore population: evaluation of new noncontact screening methods. Ophthalmology. 2008;115(10):1720-1727.

33. Liu L. Anatomical changes of the anterior chamber angle with anterior-segment optical coherence tomography. Arch Ophthalmol. 2008;126(12):1682-1686.

34. Nongpiur ME, Haaland BA, Friedman DS, et al. Classification algorithms based on anterior segment optical coherence tomography measurements for detection of angle closure. Ophthalmology. 2013;120(1):48-54.
Clinical Ophthalmology

\section{Publish your work in this journal}

Clinical Ophthalmology is an international, peer-reviewed journal covering all subspecialties within ophthalmology. Key topics include: Optometry; Visual science; Pharmacology and drug therapy in eye diseases; Basic Sciences; Primary and Secondary eye care; Patient Safety and Quality of Care Improvements. This journal is indexed on Submit your manuscript here: http://www.dovepress.com/clinical-ophthalmology-journal

\section{Dovepress}

PubMed Central and CAS, and is the official journal of The Society of Clinical Ophthalmology (SCO). The manuscript management system is completely online and includes a very quick and fair peer-review system, which is all easy to use. Visit http://www.dovepress.com/ testimonials.php to read real quotes from published authors. 